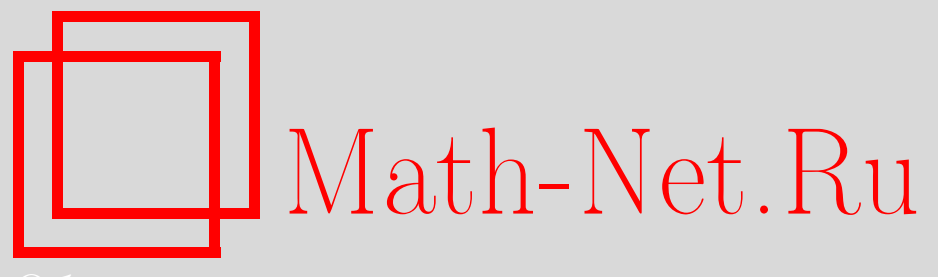

Ю. В. Бугаев, Применение прямого обобщения скалярных алгоритмов в векторной оптимизации на графах, Дискрет. матем., 2001, том 13, выпуск 3, 110-124

DOI: https://doi.org/10.4213/dm291

Использование Общероссийского математического портала Math-Net.Ru подразумевает, что вы прочитали и согласны с пользовательским соглашением http: //www . mathnet.ru/rus/agreement

Параметры загрузки:

IP : 3.93 .64 .190

26 апреля 2023 г., 12:47:11

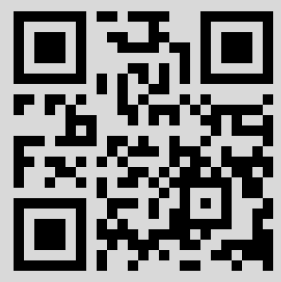




\title{
Применение прямого обобщения скалярных алгоритмов в векторной оптимизации на графах
}

\author{
(c) 2001 г. Ю. В. Бугаев
}

\begin{abstract}
Рассматривается задача поиска оптимальных путей на ориентированных графах с векторными весами дуг. В качестве критерия эффективности используется условие блокировки по бинарному отношению предпочтения, определенного на множестве путей. В качестве базового алгоритма векторной оптимизации используется схема прямого обобщения скалярных прототипов на векторный случай. Найдены достаточные условия корректности применения такого подхода.

Предлагаются два алгоритма, основанных на идее прямого обобщения, для произвольного и бесконтурного графа, доказывается их эффективность при условии асимметричности и транзитивности отношения предпочтения. Помимо этого излагается способ регулирования мощности генерируемого алгоритмами множества эффективных решений с учетом предпочтений лица, принимающего решение.
\end{abstract}

\section{1. Введение}

В процессе решения задачи векторной оптимизации на первом этапе осуществляется отбор некоторого подмножества в каком-то смысле нехудших вариантов, чаще всего - недоминируемых по Парето, а на втором этапе набор сужают до количества, обозримого лицом, принимающим решение (ЛПР), для поиска компромиссной точки с использованием неформальных процедур. Для оптимизационных задач с непрерывными переменными общеизвестны методы решения первого этапа $[1,2]$ посредством оптимизации линейной свертки

$$
L(x)=a^{T} x
$$

для выпуклых областей и критериев $x_{j} j=1, \ldots, n$, или свертки вида

$$
M(x)=\min a_{j} x_{j}
$$

для невыпуклых задач. Вектор $a=\left(a_{1}, \ldots, a_{n}\right)^{T}$ представляет собой набор весов. Кроме того, широко применяются различные варианты метода переменных ограничений $[2,3]$.

Основная идея перечисленных подходов состоит в сведении векторной задачи $\mathbf{k}$ последовательности скалярных оптимизационных задач. Нетрудно заметить, что к 
поиску эффективных решений задач дискретной оптимизации такой способ плохо приспособлен. Это связано прежде всего с отсутствием непрерывной взаимно однозначной зависимости координат эффективной точки от весовых коэффициентов $a_{j}$, $j=1, \ldots, n$, и правых частей переменных ограничений, вследствие чего для поиска всех эффективных решений необходима очень мелкая сетка на множестве варьируемых параметров. Кроме того, используемые способы скаляризации, за исключением линейной свертки, несовместимы с принципом оптимальности Беллмана, на который опираются многие алгоритмы дискретной оптимизации, в частности, методы поиска оптимальных путей в графе.

В последнее время в дискретной оптимизации ведутся активные исследования по разработке алгоритмов поиска лексикографических оптимумов $[4,5]$, а также по определению возможности применения алгоритма линейной свертки критериев (АЛСК) для получения Парето-оптимальных решений $[6,7]$. Получено подтверждение того, что АЛСК не позволяет найти все множество нехудших решений.

В [8] предложен эффективный алгоритм нахождения на графе $K$ лучших путей относительно некоторого скалярного критерия. При удачном подборе критерия, например, в виде линейной свертки, с помощью этого алгоритма можно найти множество решений из некоторой локальной области, представляющей наибольший интерес для ЛПР. Однако поиск всех нехудших путей таким методом нерационален.

Достоинство прямого обобщения скалярного алгоритма на векторный случай состоит в том, что основанные на нем методы гарантируют получение всего множества эффективных решений при небольших затратах вычислительных ресурсов. Суть его заключается в том, что в процессе поиска в множестве допустимых решений $T$ получается последовательность конечных множеств $X_{k}$ - совокупностей нехудших решений, полученных к $k$-й итерации. Множества $X_{k}$ получаются генерацией на каждом шаге некоторого набора $X_{k}^{\prime}$, который затем сужается посредством заданной функции выбора

$$
X_{k}=C\left(X_{k}^{\prime} \cup X_{k-1}\right) .
$$

При построении $X_{k}^{\prime}$ используются элементы какого-либо скалярного алгоритма. Этот подход был применен при разработке алгоритма многокритериального линейного программирования $[9,10]$. В [12] приведено доказательство результативности схемы прямого обобщения для динамического программирования. В [11] изложено обобщение алгоритма Беллмана для поиска множества путей, длина которых не превышает некоторой заданной величины.

Данная работа посвящена применению схемы прямого обобщения для поиска оптимальных путей на графе при заданных векторных весах его ребер. Предполагается, что на множестве путей определено некоторое бинарное отношение $R$ и выбор эффективных решений осуществляется посредством механизма блокировки [13]

$$
C^{R}(X)=\{x \in X: \forall y \in X(y, x) \notin R\},
$$

где $C^{R}(X)$ - результат применения функции выбора (выбор) на множестве предъявлений $X$.

\section{2. Условия корректности схемы прямого обобщения}

Очевидно, что применение схемы прямого обобщения корректно, если выполнены следующие три условия. 
(1) Условие ассоциативности выбора

$$
C\left(\bigcup_{i}^{n} X_{i}^{\prime}\right)=C\left(X_{1}^{\prime} \cup C\left(X_{2}^{\prime} \cup \ldots \cup C\left(X_{n}^{\prime}\right)\right)\right.
$$

то есть последовательный выбор на промежуточных множествах совпадает с разовым выбором на их объединении.

(2) Условие результативности генерации

$$
T^{*}=C(T) \subseteq \bigcup_{k} X_{k}^{\prime}
$$

то есть выбор на всем множестве вариантов является подмножеством сгенерированных наборов. Выполнение этого условия гарантирует, что решение задачи будет найдено.

(3) Условие эффективности перебора

$$
|T| \gg\left|\bigcup_{i}^{n} X_{i}\right|
$$

которое обеспечивает более низкую вычислительную сложность алгоритма, основанного на схеме прямого обобщения, по сравнением с полным перебором.

Ранее схема прямого обобщения использовалась для поиска оптимальных решений с выбором по Парето $[9,10,11]$. Вопрос о корректности ее применения для произвольного механизма выбора не исследовался. Определим условия соблюдения ассоциативности выбора в предлагаемой схеме.

Теорема 1. Пусть фунхиия выбора, определенная на конечном множестве $T$, обладает свойствами наследования и отбрасъвания. Тогда для любых подмножеств $X, Y \subseteq T$ справедливо равенство

$$
C(X \cup Y)=C(X \cup C(Y))
$$

Доказательство. Напомним, что свойства наследования и отбрасывания означают (см. [13]), что

$$
\begin{aligned}
(A \subseteq B) & \Rightarrow(C(B) \cap A) \subseteq C(A), \\
(C(A) \subseteq B \subseteq A) & \Rightarrow(C(B)=C(A))
\end{aligned}
$$

соответственно. Покажем, что при выполнении условий теоремы имеет место включение

$$
C(X \cup Y) \subseteq X \cup C(Y)
$$

Пусть $x \in C(X \cup Y)$. Если $x \in X$, то, очевидно, $x \in X \cup C(Y)$. Пусть теперь $x \in Y$, тогда $x \in C(X \cup Y) \cap Y$. По свойству наследования

$$
(Y \subseteq X \cup Y) \Rightarrow(C(X \cup Y) \cap Y) \subseteq C(Y) .
$$


Значит, $x \in C(Y)$, а следовательно, $x \in X \cup C(Y)$, что доказывает утверждение (2). Положим $A=X \cup Y, B=X \cup C(Y)$. По доказанному

$$
C(X \cup Y) \subseteq(X \cup C(Y)) \subseteq X \cup Y .
$$

Тогда, по свойству отбрасывания, $C(A)=C(B)$, или $C(X \cup Y)=C(X \cup C(Y))$, что и требовалось доказать.

Равенство (1) есть не что иное как запись условия ассоциативности выбора для двух множеств. По индукции оно легко обобщается на случай произвольного числа множеств. Следовательно, достаточным условием ассоциативности выбора при использовании схемы прямого обобщения является применение на каждом шаге функции выбора, обладающей свойствами наследования и отбрасывания. В частности, согласно детализации теоремы Сена [14], это имеет место, если функция выбора порождается асимметричным транзитивным бинарным отношением, то есть качественным порядком.

Пусть $X=\left\{x^{1}, \ldots, x^{N}\right\}-$ конечное множество альтернатив, на котором надо осуществить выбор и сформировать множество $P=C(X)$. Выполнение условия ассоциативности выбора позволяет решить данную задачу посредством следующего алгоритма, который, также как и другие алгоритмы, опишем на неформальной версии языка Паскаль (см. [15]):

$$
\begin{aligned}
& \text { begin } \\
& \qquad P:=\left\{x^{1}\right\} \\
& \quad \text { for } y \in X \backslash\left\{x^{1}\right\} \text { do } P:=C(P \cup\{y\}) \\
& \text { end. }
\end{aligned}
$$

Другими словами, выбор формируется последовательным сравнением альтернатив из $X$ с множеством $P$ уже отобранных вариантов.

\section{3. Векторный вариант алгоритма Форда-Беллмана}

Предлагаемый в этом разделе алгоритм $A 1$ является обобщением известного алгоритма Форда-Беллмана $[8,15]$ на случай векторного критерия эффективности. Он позволяет найти в произвольном ориентированном графе множество нехудших путей между его двумя фиксированными вершинами.

Пусть каждая дуга $[u, v]$ ориентированного графа $G$ снабжена некоторым векторным весом $A[u, v]$. Если в графе нет ребра $[u, v]$, то $A[u, v]=\infty$. Вес произвольного пути равен сумме весов составляющих дуг, и на множестве весов путей определено бинарное отношение предпочтения $R$. Точку $x, x \in X$, будем называть эффективным или $R$-оптимальным элементом множества $X$, если $x$ недоминируем по $R$ на $X$, то есть

$$
\forall y \in X, y \neq x:(y, x) \notin R .
$$

Сделаем следующие предположения.

(1) В графе $G$ каждая пара вершин может соединяться не более, чем одной дугой.

(2) Векторный вес любого контура, имеющегося в графе, доминируется нулевым вектором в смысле отношения $R$. 
(3) Отношение $R$ транзитивно, асимметрично и не зависит от смещения. Последнее означает, что для любой пары точек $(x, y)$ и любого вектора $b$ следующие отношения эквивалентны: $x R, y$ и $(x+b) R,(y+b)$. Перечисленными свойствами, в частности, обладает отношение Парето.

Введем следующие обозначения. Пусть $V$ - множество вершин графа; $\{D(v)\}$ - множество векторных весов путей от источника $s$ до вершины $v$, генерируемых алгоритмом; $\operatorname{Pr}[v]-$ список вершин графа, предшествующих вершине $v$.

Предлагается следующий алгоритм $A 1$.

$$
\begin{aligned}
& \{D(v)\}:=A[s, v] \quad \forall v \in V, v \neq s ; \quad\{D(s)\}:=\varnothing ; \\
& \text { begin } \\
& \text { for } k:=1 \text { to } n-2 \text { do } \\
& \text { for } v \in V \backslash\{s\} \text { do } \\
& \text { for } u \in \operatorname{Pr}[v] \text { do } \\
& \text { for } q \in\{D(u)\} \text { do } \\
& \qquad D(v)\}:=C^{R}(\{D(v)\} \cup(q+A[u, v]))
\end{aligned}
$$

end.

Для доказательства корректности данного алгоритма ведем понятие отношения доминирования для множеств. Пусть $R$ - некоторое бинарное транзитивное асимметричное отношение; $A$ и $B$ - некоторые множества, на элементах которых определено отношение $R$. Будем говорить, что множество $B$ доминируется множеством $A$ в смысле отношения $R$ (в этом случае будем писать $A R B$ ), если выполняется условие

$$
\forall y \in B \exists x \in A:((x, y) \in R) \vee(x=y) \text {. }
$$

Очевидно, из транзитивности $R$ вытекает транзитивность введенного отношения доминирования множеств.

Далее, допуская справедливость предположения 3 относительно бинарного отношения $R$, докажем несколько лемм.

Лемма 1. Если $A$ - конечное множество, то любой его элемент $x$, не принадлежащий выбору $C^{R}(A)$, доминируется некоторым элементом у из $C^{R}(A)$, то есть

$$
\forall x \in\left(A \backslash C^{R}(A)\right) \exists y \in C^{R}(A):(y, x) \in R .
$$

Доказательство. Предположим противное. Пусть существует такой элемент $x \in$ $A \backslash C^{R}(A)$, что для любого элемента $y \in C^{R}(A)$ отношение $(y, x) \in R$ не выполняется. Тогда либо $x \in C^{R}(A)$, что невозможно по предположению, либо найдется некоторый элемент $z_{1} \in A \backslash C^{R}(A)$ такой, что $\left(z_{1}, x\right) \in R$.

В свою очередь, элемент $z_{1}$ должен доминироваться либо некоторым $z_{2} \in A \backslash C^{R}(A)$, либо некоторым $t \in C^{R}(A)$, так как иначе должно выполняться включение $z_{1} \in C^{R}(A)$, что невозможно в силу определения $z_{1}$. Если верно второе предположение, то, в силу транзитивности $R, x$ доминируется $t$, то есть элементом $C^{R}(A)$, что невозможно. Следовательно, верно первое предположение о существовании $z_{2} \in A \backslash C^{R}(A)$ такого, что $\left(z_{2}, z_{1}\right) \in R$.

Продолжая эти рассуждения, получим последовательность элементов $z_{i} \in A \backslash C^{R}(A)$ таких, что $z_{i} R z_{i-1}$ и ни один из них не доминируется никаким 
элементом из $C^{R}(A)$, так как иначе, вследствие транзитивности $R$, получится, что $x$ доминируется элементом $C^{R}(A)$, что невозможно по предположению.

Поскольку $R$ асимметрично и транзитивно, оно и ациклично (см. [13]). Значит, в данной последовательности не может быть повторяющихся элементов. Поэтому, в силу конечности множества $A$, последовательность должна оборваться на элементе $z_{n} \in A \backslash C^{R}(A)$, для которого уже не найдется доминирующего элемента множества $A \backslash C^{R}(A)$. Но он не может доминироваться и элементом $C^{R}(A)$, так как тогда, вследствие транзитивности $R$, получится, что и $x$ доминируется этим элементом. Значит, $z_{n}$ недоминируем на $A$ и, следовательно, должен принадлежать $C^{R}(A)$, что противоречит условию $z_{n} \in A \backslash C^{R}(A)$.

Лемма 2. Пусть $A, B, D$ - конечные множества, на которых определено отношение $R$ и между $B$ u $D$ установлено отношение доминирования $B R D$. Тогда $C^{R}(A \cup B)$ доминирует $C^{R}(D)$.

Доказателъство. Пусть $y$ - произвольный элемент $C^{R}(D)$. Тогда $y \in D$. Значит, вследствие условия $B R D$, существует такой $x$ из $B$, что имеет место отношение $x R y$. Очевидно, $x \in(A \cup B)$. Если $x \in C^{R}(A \cup B)$, то для $y$ найден доминирующий элемент и лемма верна. В противном случае, в силу леммы 1 , должен существовать такой элемент $t \in C^{R}(A \cup B)$, что $t R x$. Отсюда, вследствие транзитивности $R$,

$$
(t R x \wedge x R y) \Rightarrow(t, y) \in R,
$$

что также означает существование в $C^{R}(A \cup B)$ элемента, доминирующего $y$.

Лемма 3. Если $B_{1}, D_{1}, B_{2}, D_{2}$ - конечные множества и $B_{1} R D_{1}$ u $B_{2} R D_{2}$, то $C^{R}\left(B_{1} \cup B_{2}\right)$ доминирует $C^{R}\left(D_{1} \cup D_{2}\right)$.

Доказателъство. Выберем произвольный элемент $y \in C^{R}\left(D_{1} \cup D_{2}\right)$. Пусть $y \in D_{1}$. Тогда по условию доминирования в $B_{1}$, найдется такой $x$, что $(x, y) \in R$. Если $x \in C^{R}\left(B_{1} \cup B_{2}\right)$, то имеет место доминирование $x$ над $y$. Если же $x \notin C^{R}\left(B_{1} \cup B_{2}\right)$ то по лемме 1 найдется $z \in C^{R}\left(B_{1} \cup B_{2}\right)$ такой, что $(z, x) \in R$. Отсюда, вследствие транзитивности $R,(z, y) \in R$, что также означает выполнение условия доминирования.

Если $y \in D_{2}$, то доказательство аналогично.

Лемма 3 очевидным образом обобщается на случай объединения произвольного числа пар множеств $B_{i}, D_{i}$ : если $B_{i} R D_{i}$ для всех $i$, то

$$
C^{R}\left(\bigcup_{i} B_{i}\right) R C^{R}\left(\bigcup_{i} B_{i}\right) .
$$

Лемма 4. Если $A R B, B R C$ u $A=C$, mо $A=B=C$.

Доказательство. Условие $A R B$ означает, что

$$
\forall b \in B \exists a \in A: a R b \text {. }
$$

Пусть $x \in B$. Тогда в $A$ найдется $y$, для которого $x R y$. Так как $A=C$, то $y \in C$. Это означает, что для всякого $x \in B$ найдется элемент $y \in C$ такой, что либо $y=x$, либо $y R x$. Но последнего быть не может ввиду условия $B R C$. Значит, $x=y$ и $x \in C$. C учетом равенства $A=C$ это означает, что $x \in A$ и $B \subseteq A$.

Аналогично доказывается обратное включение $A \subseteq B$. 
Лемма 5. Для любого хонечного множества $A$ выполняется соотношение

$$
C^{R}(A) R A \text {. }
$$

Доказательство. Предположим противное, то есть пусть

$$
\exists x \in A: \forall y \in C^{R}(A), x \neq y(y, x) \notin R .
$$

Тогда либо $x \in C^{R}(A)$ и, положив $y=x$, придем к противоречию, либо надо допустить, что $x \in A \backslash C^{R}(A)$. Тогда по лемме 1 должен существовать такой $y \in C^{R}(A)$, что $(y, x) \in R$, что также ведет к противоречию, доказывающему лемму.

Лемма 6. Для любых конечных множеств $A$ и $B$ выполняется соотношение

$$
C^{R}(A \cup B) R B
$$

Доказательство. Покажем, что

$$
C^{R}\left(A \cup C^{R}(B)\right) R C^{R}(B) .
$$

Положим $C^{R}(B)=D$. Тогда, по лемме $5, \quad D R B$. Отсюда по лемме 2 $C^{R}(A \cup D) R C^{R}(B)$, что эквивалентно (3).

В силу теоремы $1 C^{R}\left(A \cup C^{R}(B)\right)=C^{R}(A \cup B)$. Следовательно, $C^{R}(A \cup B) R C^{R}(B)$. Так как по лемме $5 C^{R}(B) R B$, из транзитивности отношения доминирования получаем нужное следствие.

Теперь докажем корректность алгоритма $A 1$. Имеет место следующая теорема.

Теорема 2. Если бинарное отношение $R$ и граф $G$ удовлетворяют предположениям 1-3, то множество решений, полученных с помощью алгоритма $A 1$, совпадает с множеством решений, недоминируемых в смысле отношения $R$.

Доказательство. Пусть $\rho(Y)$ - векторный вес пути $Y,\left\{D^{(m)}(v)\right\}$ - множество, элементы которого $D^{(m)}(v)$ суть веса всех путей из $s$ в $v$, содержащих не более $m$ дуг, $\left\{d^{(m)}(v)\right\}$ - множество весов $d^{(m)}(v)$ всех путей из $s$ в $v$, содержащих не более $m$ дуг и недоминируемых на множестве $\left\{D^{(m)}(v)\right\}$ в смысле отношения $R$, то есть

$$
\left\{d^{(m)}(v)\right\}=C^{R}\left\{D^{(m)}(v)\right\} .
$$

Докажем, что

$$
\forall m>0, v \in V\left\{d^{(m+1)}(v)\right\}=C^{R}\left(\bigcup_{u \in \operatorname{Pr}[v]}\left\{d^{(m)}(u)+A[u, v]\right\}\right)
$$

Пусть $X$ - некоторый путь из $s$ в $v$, состоящий не более чем из $m+1$ дуг и недоминируемый на $\left\{D^{(m+1)}(v)\right\}$. Пусть $u$ - предпоследняя вершина пути $X$, а $X^{\prime}$ - его часть от $s$ до $u$, то есть $X=X^{\prime} \cup[u, v]$. Тогда $\rho\left(X^{\prime}\right) \in\left\{d^{(m)}(u)\right\}$.

Предположим, что это не так, то есть

$$
\rho\left(X^{\prime}\right) \in\left\{D^{(m)}(u)\right\} \backslash\left\{d^{(m)}(u)\right\} .
$$


Тогда по лемме 1 должен существовать доминирующий $\rho\left(X^{\prime}\right)$ элемент, то есть найдется путь $Y^{\prime}$ такой, что $\rho\left(Y^{\prime}\right) \in\left\{d^{(m)}(u)\right\}$, и имеет место отношение $\rho\left(Y^{\prime}\right) R \rho\left(X^{\prime}\right)$. Тогда для пути $Y=Y^{\prime} \cup[u, v]$ выполняется отношение $\rho(Y) R \rho(X)$. Это вытекает из того, что граф не содержит кратных дуг (а значит, конечные дуги путей $\mathrm{X}$ и $\mathrm{Y}$ совпадают), а также из того, что

$$
\rho(Y)=\rho\left(Y^{\prime}\right)+A[u, v], \quad \rho(X)=\rho\left(X^{\prime}\right)+A[u, v]
$$

и $R$ не зависит от смещения $A[u, v]$.

Такого пути $Y$, состоящего не более чем из $m+1$ дуг и лучшего по $R$, чем $X$, по предположению быть не должно. Значит, верно, что $\rho\left(X^{\prime}\right) \in\left\{d^{(m)}(u)\right\}$.

Следовательно, $\rho(X) \in\left\{d^{(m)}(u)+A[u, v]\right\}$, а отсюда,

$$
\rho(X) \in \bigcup_{u \in \operatorname{Pr}[v]}\left\{d^{(m)}(u)+A[u, v]\right\}
$$

Введем обозначения

$$
F=\bigcup_{u \in \operatorname{Pr}[v]}\left\{d^{(m)}(u)+A[u, v]\right\}, \quad H=\left\{D^{(m+1)}(v)\right\}
$$

Очевидно, что $F \subseteq H$.

Только что было доказано, что если $\rho(X) \in C^{R}(H)$ то $\rho(X) \in F$. Следовательно, $C^{R}(H) \subseteq F$. Поскольку отношение $R$ является качественным порядком, порожденная им функция выбора обладает свойством отбрасывания [13], то есть из $C^{R}(H) \subseteq F \subseteq H$ следует

$$
C^{R}(H)=C^{R}(F)
$$

Равенство (5) - это не что иное, как соотношение (4). Следовательно, (4) доказано.

Докажем следующее утверждение: если перед переходом к очередной $k$-й итерации внешнего цикла алгоритма выполняется соотношение

$$
\forall v \in V \backslash\{s\} \quad\{D(v)\} R\left\{d^{(k)}(v)\right\},
$$

то по окончании итерации справедливо включение

$$
\forall v \in V \backslash\{s\} \quad\{D(v)\} R\left\{d^{(k+1)}(v)\right\} .
$$

Пусть при некотором $k$ выполняется отношение (6). Обозначим $\left\{D_{b}(v)\right\}$ и $\left\{D_{e}(v)\right\}$ содержимое множества $\{D(v)\}$ перед и после очередной итерации внешнего цикла, соответственно, и $\left\{D_{i}(v)\right\}$ - текущее содержимое $\{D(v)\}$ внутри цикла.

Цикл for $q \in\{D(u)\}$ алгоритма реализует операцию пошагового построения множества значений функции выбора непосредственно после рассмотрения $\{D(u)\}$, соответствующего каждой очередной вершине $u \in \operatorname{Pr}[v]$. Но согласно обобщению теоремы 1 такое построение можно проводить после рассмотрения всего набора $\operatorname{Pr}[v]$ вершин, предшествующих $v$. Этот факт позволяет представить $D_{e}(v)$ в виде

$$
\left\{D_{e}(v)\right\}=C^{R}\left(\bigcup_{u \in \operatorname{Pr}[v]}\left\{D_{i}(u)+A[u, v]\right\} \bigcup\left\{D_{b}(v)\right\}\right)
$$


В зависимости от очередности рассмотрения вершин в цикле for $v \in V \backslash\{s\}$, все элементы множества $\left\{D_{i}(u)\right\}$ могут совпадать либо с $\left\{D_{b}(u)\right\}$, либо с $\left\{D_{e}(u)\right\}$. Пусть для какой-то вершины $u \in V \backslash\{s\}$ имеет место равенство $\left\{D_{i}(u)\right\}=\left\{D_{b}(u)\right\}$. Тогда по предположению об истинности отношения (6) $\left\{D_{i}(u)\right\} R\left\{d^{(k)}(u)\right\}$.

Если же для какой-то вершины $u$ выполняется равенство $\left\{D_{i}(u)\right\}=\left\{D_{e}(u)\right\}$, то в силу (8)

$$
\left\{D_{e}(u)\right\}=C^{R}\left(M \cup\left\{D_{b}(u)\right\}\right),
$$

где

$$
M=\bigcup_{w \in \operatorname{Pr}[u]}\left\{D_{i}(w)+A[w, u]\right\}
$$

По предположению $\left\{D_{b}(u)\right\} R\left\{d^{(k)}(u)\right\}$, поэтому согласно лемме 2 выполняется отношение

$$
C^{R}\left(M \cup\left\{D_{b}(u)\right\}\right) R C^{R}\left(\left\{d^{(k)}(u)\right\}\right) .
$$

Поскольку $\left\{d^{(k)}(u)\right\}$ состоит только из недоминируемых элементов множества $\left\{D^{(k)}(u)\right\}$, то $C^{R}\left(\left\{d^{(k)}(u)\right\}=\left\{d^{(k)}(u)\right\}\right.$ и получаем, что $\left\{D_{e}(u)\right\} R\left\{d^{(k)}(u)\right\}$.

Таким образом, как при $\left\{D_{i}\right\}=\left\{D_{b}\right\}$, так и при $\left\{D_{i}\right\}=\left\{D_{e}\right\}$ имеет место отношение $\left\{D_{i}(u)\right\} R\left\{d^{(k)}(u)\right\}$. Поскольку из независимости $R$ от смещения вытекает наличие этого свойства и для отношения множественного доминирования, последнее отношение не нарушится, если к каждому элементу обоих множеств прибавить $A[u, v]$. Следовательно,

$$
\forall u \in V \backslash\{s\} \quad\left\{D_{i}(u)+A[u, v]\right\} R\left\{d^{(k)}(u)+A[u, v]\right\} .
$$

Отсюда по обобщению леммы 3 следует, что

$$
C^{R}\left(\bigcup_{u \in \operatorname{Pr}[v]}\left\{D_{i}(u)+A[u, v]\right\}\right) R C^{R}\left(\bigcup_{u \in \operatorname{Pr}[v]}\left\{d^{(k)}(u)+A[u, v]\right\}\right)
$$

а в силу леммы 2 отношение доминирования не нарушится, если аргумент левого операнда объединить с произвольным множеством, например с $\left\{D_{b}(v)\right\}$. После такого объединения получаем, что

$$
C^{R}\left(\bigcup_{u \in \operatorname{Pr}[v]}\left\{D_{i}(u)+A[u, v]\right\} \cup\left\{D_{b}(v)\right\}\right) R C^{R}\left(\bigcup_{u \in \operatorname{Pr}[v]}\left\{d^{(k)}(u)+A[u, v]\right\}\right)
$$

Левая часть (9) согласно (8) есть не что иное как $\left\{D_{e}(v)\right\}$, а правая в силу (4) есть $\left\{d^{(k+1)}(v)\right\}$. Отсюда следует справедливость условия (7).

Так как перед первой итерацией внешнего цикла выполнено равенство

$$
\forall v \in V \backslash\{s\}\left\{D_{b}(v)\right\}=\left\{d^{(1)}(v)\right\}
$$

по индукции (7) выполняется после всех итераций, а значит, после окончания цикла (когда $k=n-2$ ) справедливо отношение

$$
\{D(v)\} R\left\{d^{(n-1)}(v)\right\}
$$

Обозначим через $d(s, v)$ множество недоминируемых по $R$ весов путей из $s$ в $v$, состоящих из произвольного числа дуг. Тогда по лемме 5 выполняется отношение 
$d(s, v) R\{D(v)\}$. Но $d(s, v)=\left\{d^{(n-1)}(v)\right\}$, так как каждый путь с более чем $n-1$ дугами содержит контур, устранение которого, в силу предположения 2 , может сделать путь только более предпочтительным в смысле отношения $R$. Отсюда, по лемме 4

$$
\{D(v)\}=d(s, v)
$$

что и требовалось доказать.

Замечание 1. Справедливость предположения 2 легко проверяется средствами самого алгоритма так же, как и в скалярном случае [8]. Для этого после каждого обновления множества $\{D(v)\}$ и нахождения вершины $u^{*}$, соответствующей новому эффективному пути, необходимо проверить принадлежность вершины $v$ текущему эффективному пути от $s$ до $u^{*}$. Если принадлежность имеет место, то $u^{*}$ была пройдена через промежуточную вершину $v$. Следовательно, часть эффективного пути от $v$ до $u^{*}$ вместе с дугой $\left[u^{*}, v\right]$ образуют контур, вес которого предпочтительнее нулевого вектора. Если же принадлежность не подтверждается ни на одной итерации, то такого контура нет.

\section{4. Поиск эффективных путей в бесконтурном графе}

В случае отсутствия в графе $G$ контуров имеется более экономный алгоритм $A 2$, скалярный прототип которого описан в $[8,15]$. Предполагается, что вершины графа пронумерованы так, что для любой дуги $\left[v_{i}, v_{j}\right]$ выполняется соотношение $i<j$. В [15] показано, что такую нумерацию можно установить в любом бесконтурном графе.

Пусть $\left\{v_{1}, \ldots, v_{n}\right\}$ - множество вершин графа, пронумерованных указанным способом, $v_{1}$ и $v_{n}$ - начальная и конечная вершины, соответственно. Предлагается следующий алгоритм $A 2$.

$$
\begin{aligned}
& D\left[v_{i}\right]:=A\left[v_{1}, v_{i}\right], i>1 ; \quad D\left[v_{1}\right]:=0 \\
& \text { begin } \\
& \text { for } i:=2 \text { to } n \text { do } \\
& \text { for } u \in \operatorname{Pr}\left[v_{i}\right] \text { do } \\
& \text { for } q \in\{D[u]\} \text { do } \\
& \left.\qquad D\left[v_{i}\right]\right\}:=C^{R}\left(\left\{D\left[v_{i}\right]\right\} \cup\left(q+A\left[u, v_{i}\right]\right)\right)
\end{aligned}
$$

end.

Корректность алгоритма устанавливает следующая теорема.

Теорема 3. Если отношение предпочтения $R$ и бесконтурный граф $G$ удовлетворяют предположениям 1-3, то множество решений, найденных алгоритмом $A 2$, совпадает с множеством путей, недоминируемых по $R$.

Доказательство. Прежде всего в силу теоремы 1 множества $\left\{D\left(v_{i}\right)\right\}$, которые генерирует алгоритм, можно представить в виде

$$
\left\{D\left(v_{i}\right)\right\}=C^{R}\left(\bigcup_{u \in \operatorname{Pr}\left[v_{i}\right]}\left\{D(u)+A\left[u, v_{i}\right]\right\}\right)
$$


Покажем, что веса всех эффективных в смысле отношения $R$ путей из $v_{1}$ в $v_{n}$ принадлежат множеству $\left\{D\left(v_{n}\right)\right\}$.

Предположим противное. Пусть нашелся эффективный путь $P$ из $v_{1}$ в $v_{n}$, вес которого не принадлежит $\left\{D\left(v_{n}\right)\right\}$. Пусть $u$ - его предпоследняя вершина. Тогда $P^{\prime}=P \backslash\left[u, v_{n}\right]$ является эффективным путем из $v_{1}$ в $u$. В противном случае, по лемме 1 существует путь $Q^{\prime}$ из $v_{1}$ в $u$, вес которого предпочтительнее веса $P^{\prime}$ по отношению $R$. Тогда путь $Q=Q^{\prime} \cup\left[u, v_{n}\right]$ доминирует путь $P$, что невозможно по определению $P$.

Для пути $P^{\prime}$ может выполняться одно из соотношений

$$
\begin{aligned}
& \rho\left(P^{\prime}\right) \in\{D(u)\}, \\
& \rho\left(P^{\prime}\right) \notin\{D(u)\} .
\end{aligned}
$$

Если верно первое соотношение, то $\rho(P) \in \cup\left\{D(u)+A\left[u, v_{n}\right]\right\}$ и, значит, по лемме 1 путь $P$ обязательно будет выбран алгоритмом, и тогда $\rho(P) \in\left\{D\left(v_{n}\right)\right\}$, что противоречит сделанному предположению.

Если верно второе соотношение, то рассмотрим путь $P^{\prime \prime}=P^{\prime} \backslash[w, u]$, где $w-$ предпоследняя вершина пути $P^{\prime}$. Для $P^{\prime \prime}$ также может выполняться одно из соотношений $\rho\left(P^{\prime \prime}\right) \in\{D(w)\}$ и $\rho\left(P^{\prime \prime}\right) \notin\{D(w)\}$. Выполнение первого соотношения, как и для пути $P^{\prime}$, приводит к противоречию.

Продолжая рассуждения, придем к пути $P^{k}$, состоящему из одной дуги $\left[v_{1}, r\right]$, вес которого по предположению не может принадлежать $\{D(r)\}$. С другой стороны,

$$
\rho\left(P^{k}\right) \in \bigcup_{x \in \operatorname{Pr}[r]}\{D(x)+A[x, r]\},
$$

поскольку вершина $v_{1}$ является предшествующей для $r$. Значит, по лемме 1 путь $P^{k}$ будет выбран алгоритмом и, следовательно, $\rho\left(P^{k}\right) \in\{D(r)\}$. Полученное противоречие доказывает принадлежность веса эффективного пути $P$ множеству $\left\{D\left(v_{n}\right)\right\}$.

Обратное включение очевидно, поскольку если какое-то решение не является эффективным, то есть не принадлежит результирующему выбору, то оно обязательно отсеется на каком-то промежуточном этапе.

\section{5. Вычислительная сложность алгоритмов. Методы регулирования мощности множества эффективных решений}

Оценим вычислительную сложность алгоритмов $A 1$ и $A 2$. Пусть $L=\max |d(s, v)|$, $k$ - число критериев, $n$ - число вершин графа. При каждом повторении цикла for $q \in\{D[u\}$ необходимо не более $L$ раз провести парное сравнение $k$-мерных точек. Следовательно, вычислительная сложность выполнения всего цикла составит $O\left(k L^{2}\right)$. Поскольку в практически значимых задачах $k$ ограничено некоторой постоянной, можно считать вычислительную сложность внутреннего цикла равной $O\left(L^{2}\right)$.

Число повторений каждого из трех внешних циклов алгоритма $A 1$ имеет порядок $O(n)$, следовательно, алгоритм $A 1$ имеет вычислительную сложность $O\left(L^{2} n^{3}\right)$. Напомним, что вычислительная сложность скалярного алгоритма Форда-Беллмана составляет $O\left(n^{3}\right)$ (см. [15]). 
Аналогично можно показать, что вычислительная сложность алгоритма $A 2$ coставляет $O\left(L^{2} n^{2}\right)$ против $O\left(n^{2}\right)$ у скалярного прототипа (см. [15]).

Дать точную оценку величины $L$ для произвольного графа весьма сложно. Известны работы, в которых предлагаются такие оценки для некоторых классов задач, например, двухкритериальных [16]. Можно привести пример редкого графа, в котором все допустимые пути являются Парето-оптимальными.

Таким образом, при поиске оптимальных путей на графах с большим числом дуг мощность множества эффективных решений может достигать очень большой величины, вследствие чего резко возрастает вычислительная сложность алгоритмов. Это можно преодолеть, усилив избирательность выбора на каждой итерации. Чтобы при этом не потерять оптимальных решений, выбор следует строить с учетом предпочтений ЛПР.

В [12] описан метод идентификации системы предпочтения ЛПР, названный экстраполяцией экспертных оценок. Суть его состоит в следующем. Делается два предположения.

(4) Существует линейная по критериям функция полезности, реализующая бинарное отношение предпочтения ЛПР $R_{U}$, то есть такая скаліярная функция $U(x)$, что

$$
(x, y) \in R_{U} \Longleftrightarrow U(x) \leqslant U(y) .
$$

Условия существования такой линейной функции указаны в [13].

(5) ЛПР способен из любой пары альтернатив $x$ и $y$ выбрать лучшую по полезности.

В случае линейной функции полезности неравенство $U(x) \leqslant U(y)$ приобретает вид линейного неравенства

$$
a^{T}(x-y) \leqslant 0
$$

где $a-$ неизвестный вектор коэффициентов, координаты которого соответствуют относительной значимости частных критериев задачи. Поскольку согласно данному определению веса $a_{j}$ могут быть определены с точностью до постоянного множителя [13], необходимо ввести условие нормировки, например $\sum a_{j}=1$. Кроме того, предполагая, что все частные критерии необходимо минимизировать, получаем условие $a_{j} \geqslant 0$ для всех $j$.

Предъявляя ЛПР ограниченный набор выбранных пар альтернатив $x^{i}, y^{i}$, $i=1, \ldots, m$, для ранжирования, можно построить систему линейных условий вида

$$
\begin{aligned}
a^{T}\left(x^{i}-y^{i}\right) & \leqslant 0, i=1, \ldots, m, \\
\sum a_{j} & =1, \\
a_{j} & \geqslant 0
\end{aligned}
$$

которым должны удовлетворять коэффициенты $a_{j}$. В (11) предполагается, что с точки зрения ЛПР для всех $i$ альтернатива $x^{i}$ лучше, чем $y^{2}$.

Применительно к задаче поиска эффективных путей на графе в качестве ранжируемых альтернатив могут выступать пути, найденные каким-либо простым способом, например, с помощью АЛСК. Число точек, предъявляемых для экспертизы, может быть небольшим, в пределах десятка [17]. 
Способ уменьшения количества решений с учетом предпочтений ЛПР состоит в построении на основании системы (11) нового бинарного отношения $R_{1}$, промежуточного по избирательной способности между $R_{U}$ и отношением Парето. Обозначим $A$ множество весов $a$, удовлетворяющих системе (11). Введем отношение $R_{1}$, полагая

$$
(x, y) \in R_{1} \Longleftrightarrow\left(\forall a \in A a^{T}(x-y) \leqslant 0\right) \wedge\left(\exists a_{0} \in A: a_{0}^{T}(x-y)<0\right) .
$$

Его свойства определяются следующей теоремой.

Теорема 4. Отношение $R_{1}$, задаваемое (12), удовлетворяет условиям теорем 2, 3 .

Доказательство. Докажем асимметричность $R_{1}$. Пусть для пары $(x, y)$ выполняется отношение $(x, y) \in R_{1}$. Тогда в силу условия $a^{T}(x-y) \leqslant 0$ для всех $a \in A$ в множестве $A$ нет вектора $a_{0}$, для которого справедливо неравенство $a_{0}^{T}(y-x)<0$. Следовательно, обратное отношение $(y, x) \in R_{1}$ не будет иметь места и, значит, $R_{1}$ асимметрично.

Докажем транзитивность. Условие (12) можно представить в виде

$$
(x, y) \in R_{1} \Longleftrightarrow\left\{\begin{array}{l}
\max a^{T}(x-y) \leqslant 0, \\
\min a^{T}(x-y)<0 .
\end{array}\right.
$$

Пусть для альтернатив $x, y, z$ выполняются отношения

$$
(x, y) \in R_{1}, \quad(y, z) \in R_{1} .
$$

Это означает, что

$$
\left(\max a^{T}(x-y) \leqslant 0\right) \wedge\left(\max a^{T}(y-z) \leqslant 0\right) .
$$

Фиксируем произвольный вектор $a \in A$ и определим знак выражения

$$
a^{T}(x-z)=a^{T}(x-y)+a^{T}(y-z) .
$$

Поскольку максимум каждого слагаемого в правой части (14) неположителен, при любом $a \in A$ их сумма также не может быть строго больше нуля. Это означает, что

$$
\forall a \in A \quad a^{T}(x-z) \leqslant 0 .
$$

Кроме того, минимум каждого слагаемого в правой части (14) строго отрицателен. Совместно с (15) это означает, что при совпадении их точек минимума существует по крайней мере один вектор $a_{0}$, при котором $a_{0}^{T}(x-z)<0$, а при несовпадении минимумов таких векторов по крайней мере два. Следовательно, выполняется отношение $(x, z) \in R_{1}$, что доказывает транзитивность $R_{1}$.

Условие независимости $R_{1}$ от смещения с очевидностью вытекает из линейности выражений (12). Теорема доказана.

Для построения функции выбора на основе отношения $R_{1}$ необходимо для каждой пары сравниваемых альтернатив решать задачу линейного программирования. Более эффективный алгоритм сравнения можно построить, если воспользоваться свойством качественного порядка [13].

Согласно этому свойству, качественный порядок можно представить как отношение Парето на некотором наборе критериальных функций. Очевидно, что как 
минимум, так и максимум линейной функции $a^{T}(x-y)$ достигаются в базисных точках $a^{i}$ системы (11), координаты которых могут быть использованы в качестве коэффициентов искомых линейных критериальных функций $f_{i}(x)=x^{T} a^{i}$, и отношение $R_{1}$ можно представить в виде

$$
(x, y) \in R_{1} \Longleftrightarrow \forall i f_{i}(y)-f_{i}(x) \geqslant 0 \wedge \exists j: f_{j}(y)-f_{j}(x)>0 .
$$

Автором разработан алгоритм получения базисных точек системы вида (11) сложности $O\left(m^{k}\right)$ ( $m$ - число ранжируемых пар альтернатив, $k$ - число критериев), который удовлетворительно работает при размерности системы, лимитируемой ранжирующими способностями ЛПР, то есть при $k \leqslant 7, m \leqslant 10$ (см. $[12,17])$.

Покажем, что оптимальные по полезности альтернативы будут отобраны при использовании отношения $R_{1}$ для построения функции выбора.

Лемма 7. Пусть $a_{1} \in A$ - некоторый фиксированный вектор, удовлетворяющий системе (11). Пусть $q$ пробегает множество альтернатив $X$ и минимум линейной функции $a_{1}^{T} q$ достигается в единственной точке $q=x$. Тогда алътернатива $x$ сохранится в множестве эффективных решений при выборе посредством механизма блокировки, определяемого отношением $R_{1}$ (то естъ при выборе алътернатив, недоминируемых в смысле отношения $R_{1}$ ).

Доказательство. Согласно определению $R_{1}$ точка $x$ будет включена в выбор, если

$$
\forall y \in X\left(\exists a \in A: a^{T} x<a^{T} y\right) \vee\left(\forall a \in A: a^{T} x \leqslant a^{T} y\right)
$$

Так как $x$ - единственная точка минимума функции $a_{1}^{T} q$, при $a=a_{1}$ выполняется условие $a^{T} x<a^{T} y$ для всех $y \in X$ и, следовательно, $x$ будет включена в выбор.

Теорема 5. Хотя бы одно оптимальное по полезности решение сохраняется при выборе посредством механизма блокировки по $R_{1}$.

Доказательство. Пусть $a_{0}-$ вектор истинных значений коэффициентов линейной функции полезности. Покажем, что $a_{0} \in A$. Возьмем любую пару $x^{i}, y^{i}$, составляющих систему (11). Если бы при $a=a_{0}$ условие $a^{T}\left(x^{i}-y^{i}\right) \leqslant 0$ не выполнялось, то ЛПР не предпочел бы в этой паре точку $x^{i}$. Следовательно, $a_{0}$ удовлетворяет системе (11) и значит, принадлежит $A$.

Если $x$ - единственная оптимальная по полезности альтернатива, то для вектора $a_{0}$ и точки $x$ выполнены условия леммы 7 и $x$ будет включена в выбор.

Предположим теперь, что имеется некоторое множество $X^{\prime}$ оптимальных по полезности альтернатив таких, что

$$
\forall y \in X \backslash X^{\prime}, \quad \forall x \in X^{\prime} \quad a_{0}^{T} x<a_{0}^{T} y .
$$

Пусть $x^{1}$ - первая по порядку альтернатива из $X^{\prime}$. Тогда при реализации алгоритма последовательного выбора точка $x^{1}$ первоначально будет сравниваться только с элементами множества $X \backslash X^{\prime}$ и в силу (16) попадет в текущий выбор. При дальнейших сравнениях она может быть исключена только после сравнения с некоторой альтернативой $x^{j} \in X^{\prime}$. В этом случае $x^{j}$ включается в выбор и попадает в те же условия, что и $x^{1}$. Таким образом, хотя бы одна альтернатива из множества оптимальных по полезности вариантов обязательно будет включена в выбор. Теорема доказана. 


\section{Список литературы}

1. Гермейер Ю. В., Введение в теорию исследования операций. Наука, Москва, 1971.

2. Подиновский В. В., Ногин В. Д., Парето-оптималъные решения многокритериалъных задач. Наука, Москва, 1982.

3. Бенайюн Р., Ларичев О. И., Линейное программирование при многих критериях: метод ограничений. Автоматика и телемех. (1971), Ne8, 24-31.

4. Емеличев В. А., Гирлих Э., Янушкевич О. А., Лексикографические оптимумы многокритериальных задач. Дискретный анализ и исследование операчий (1997) 4, №2, 3-17.

5. Ivanchev D., Kudros D., Multicriteria optimum path problems. Yugosl. J. Oper. Res. (1995) 5, №1, 79-93.

6. Меламед И. И., Сигал И. Х., Теория и алгоритмъ решения многокритериалънъх задач комбияаторной оптимизачии. Изд-во ВЦ РАН, Москва, 1996.

7. Кравцов М. К., Неразрешимость задач векторной дискретной оптимизации в классе алгоритмов линейной свертки критериев. Дискретная математика (1996) 8, №2, 8996.

8. Кристофидес Н., Теория графов. Алгоритмический подход. Мир, Москва, 1978.

9. Zeleny M., Linear multiobjective programming. Springer, Berlin, 1974.

10. Yu P. L., Zeleny M., The set of all nondominated solutions in linear cases and a multicriteria simplex method. J. Math. Anal. and Appl. (1975) 49, 450-468.

11. Kremar-Nozic E., Visekriterijumsko odredivanje nedominiranih puteva izmedu zadatih cvorova u mrezi-uopstrnje Bellmanovog algoritma. Nauc. Tehn. Pregl. VTI (1984) 34, №5, 22-24.

12. Сысоев В. В., Структурные и алгоритмические модели автоматизированного проехтирования производства изделий электронной техники. Воронеж. технол. ин-т, Воронеж, 1993.

13. Юдин Д. Б., Вычислителъные методъ теории прияятия решений. Наука, Москва, 1989.

14. Айзерман М. А., Некоторые новые задачи общей теории выбора. Обзор одного направления исследования. Автоматиха и телемех. (1984), №9, 5-43.

15. Липский В., Комбинаторика для программистов. Мир, Москва, 1988.

16. Козін І. В., Про оцінки потуженості повноі множини альтернатив для деяких двукритеріальних задач на графах. Докл. АН Укр. (1993), №10, 108-111.

17. Емельянов С. В., Ларичев О. И., Многохритериалънъе методы принятия решений. Знание, Москва, 1985.

Статья поступила 11.02.1999.

Исправленный вариант поступил 22.03.2000. 